\title{
Window of opportunity for surgical decompression in patients with acute traumatic cervical spinal cord injury
}

\author{
Marko Jug, MD, PhD, ${ }^{1}$ Nataša Kejžar, PhD, ${ }^{2}$ Matej Cimerman, MD, $\mathrm{PhD},{ }^{1}$ and \\ Fajko F. Bajrović, MD, PhD ${ }^{3,4}$
}

\begin{abstract}
1Department of Traumatology and ${ }^{3}$ Neurology Clinic, University Medical Centre Ljubljana; and ${ }^{2}$ Institute for Biostatistics and Medical Informatics and ${ }^{4}$ Institute of Pathophysiology, University of Ljubljana, Faculty of Medicine, Ljubljana, Slovenia
\end{abstract}

\begin{abstract}
OBJECTIVE The objective of this prospective study was to determine the optimal timing for surgical decompression $(\mathrm{SD})$ in patients with acute traumatic cervical spinal cord injury $(\mathrm{tSCl})$ within the first 24 hours of injury.

METHODS In successive patients with fracture and/or dislocation of the subaxial cervical spine and American Spinal Injury Association Impairment Scale (AIS) grades A-C, receiver operating characteristic curve analysis was used to determine the optimal timing for SD within the first 24 hours of cervical tSCI to obtain a neurological recovery of at least two AIS grades. Multivariate logistic regression was used to model significant neurological recovery with time to SD, degree of spinal canal compromise (SCC), and severity of injury.
\end{abstract}

RESULTS In this cohort of 64 patients, the optimal timing for SD to obtain a significant neurological improvement was within 4 hours of injury (95\% confidence interval 4-9 hours). Increasing the delay from injury to SD or the degree of SCC significantly reduced the likelihood of significant neurological improvement. Due to the strong correlation with SCC, the severity of injury was a marginally significant predictor of neurological recovery.

CONCLUSIONS These findings indicate that in patients with acute cervical tSCl and AIS grades A-C, the optimal timing for SD is within the first 4-9 hours of injury, depending on the degree of SCC and the severity of injury. Further studies are required to better understand the interrelationships among the timing of SD, injury severity, and degree of SCC in these patients.

https://thejns.org/doi/abs/10.3171/2019.10.SPINE19888

KEYWORDS spinal cord injury; surgical timing; surgical decompression; injury severity; spinal canal compromise

\begin{abstract}
$\Lambda$ CUTE traumatic spinal cord injury (tSCI) is a distressing event that often leaves patients with deep and lasting motor, sensory, and autonomic incapacity. ${ }^{7}$ Currently suggested treatment modalities to improve neurological outcome in patients with tSCI include early surgical decompression (SD), blood pressure augmentation, and, optionally, methylprednisolone administration. ${ }^{3}$ Based on the results of a single prospective cohort study ${ }^{14}$ most recent clinical practice guidelines for adult patients with acute tSCI suggest that SD within 24 hours of injury should be considered. ${ }^{13}$ However, the authors also proposed additional research to explore the effectiveness of surgery within earlier time frames (such as 12 hours). ${ }^{13}$ Recently, two studies were published investigating the ef-
\end{abstract}

fect of SD on neurological outcome within 5 or 8 hours after acute cervical tSCI and reported conflicting results. ${ }^{18,20}$ In one study, neurological recovery was superior with SD within 8 hours of injury compared to SD within 8-24 hours. ${ }^{18}$ In another study, neurological recovery was inferior with SD within the first 5 hours of injury compared to SD within 5-24 hours. ${ }^{20}$ It is notable that both studies investigated the effect of the timing of SD within 24 hours by comparing patient subgroups in which the time cutoff point was arbitrarily preset at 5 or 8 hours after injury and not by searching for the optimal time cutoff point within the first 24 hours of injury. Therefore, this optimal time cutoff point remains unknown. The objective of this study was to use a receiver operating characteristic (ROC) curve

ABBREVIATIONS AIS = ASIA Impairment Scale; ASIA = American Spinal Injury Association; $C I$ = confidence interval; ICU = intensive care unit; IQR = interquartile range; $\mathrm{NASCIS}=$ National Acute Spinal Cord Injury Study; ROC = receiver operating characteristic; $\mathrm{SCC}=$ spinal canal compromise; $\mathrm{SD}=\mathrm{surgical}$ decompression; $\mathrm{tSCI}=$ traumatic spinal cord injury.

SUBMITTED July 29, 2019. ACCEPTED October 8, 2019.

INCLUDE WHEN CITING Published online December 27, 2019; DOI: 10.3171/2019.10.SPINE19888. 
TABLE 1. Inclusion and exclusion criteria

\begin{tabular}{cr}
\hline Inclusion Criteria & Exclusion Criteria \\
\hline Age 16-85 yrs & Neurological deficits prior to injury \\
\hline Fracture or dislocation level C3-7 & No evidence of fracture or dislocation \\
\hline Spinal cord compression confirmed by MRI & Life-threatening injuries that prevent early decompression of the spinal cord \\
\hline Neurological level of injury between C3 \& C8 & Cognitive impairment preventing accurate neurological assessment \\
\hline Initial AlS grades A-C & \\
\hline SCC of at least $25 \%$ & \\
\hline Surgery within 24 hrs of tSCl & \\
\hline
\end{tabular}

to evaluate the impact of timing of SD and instrumented fusion within the first 24 hours of injury on significant neurological recovery (at least two American Spinal Injury Association [ASIA] Impairment Scale [AIS] grades), to determine the optimal time window for SD in patients with acute cervical tSCI and persistent compression of the spinal cord. In addition, we evaluated the association of timing of SD adjusted by the degree of spinal canal compromise (SCC) and the severity of cervical tSCI (complete or incomplete).

\section{Methods \\ Study Population}

A prospective cohort study was conducted in successive patients with acute cervical tSCI and fracture or dislocation of the subaxial cervical spine operated on within the first 24 hours of injury from January 2007 to December 2016 at a single level 1 trauma center (UMC Ljubljana, Slovenia). Approval of the Research Ethics Board was obtained before patient enrollment. Written informed consent was not acquired as prior to analysis patient data were anonymized.

A set of inclusion and exclusion criteria were defined prior to enrollment (Table 1). As suggested by the Sygen trial, ${ }^{16}$ to permit a potential improvement of two AIS grades, only patients with overall AIS grades $\mathrm{A}-\mathrm{C}^{19}$ were enrolled. The neurological examination was performed according to the International Standards for Neurological Classification of Spinal Cord Injury ${ }^{19}$ immediately after admission to the emergency room and at the end of rehabilitation 6 months after injury by a qualified orthopedic trauma specialist who was blinded with respect to timing of surgery. After initial stabilization in the emergency room all patients underwent $\mathrm{CT}$ and MRI of their cervical spine. The degree of SCC was assessed on a midsagittal T2-weighted MR image ${ }^{12}$ and after surgery reassessed with CT. According to the treatment protocol all patients underwent SD in the operating room "as soon as possible" after stabilization and imaging. The time of SD was defined as the time from injury to successful decompression of the spinal cord by means of closed or open reduction of spinal dislocations and/or direct decompression of the spinal cord, immediately followed by spinal instrumentation in all patients. All patients were given methylprednisolone (Solu-Medrol, Pfizer Luxembourg SARL) according to recommendations of the third National Acute Spinal
Cord Injury Study (NASCIS) ${ }^{5,6}$ and admitted to the same intensive care unit (ICU) for optimal postoperative care, including monitoring and control of mean arterial blood pressure at a goal level of $85 \mathrm{~mm} \mathrm{Hg}$. All patients were moved to the same clinic for rehabilitation after cardiopulmonary stabilization.

\section{Outcome}

The primary outcome of interest was the effect of the timing of SD within the first 24 hours of injury on the ordinal change in the grade of AIS at the 6-month followup. A follow-up period of 6 months was based on recommendations used in the Sygen trial and the NASCIS. 5,16 Furthermore, the effect on neurological recovery of the degree of SCC and the severity of cervical tSCI (complete vs incomplete) was evaluated. Significant neurological recovery was defined as an improvement of at least two AIS grades, the severity of cervical tSCI as a complete cervical tSCI group (AIS grade A) or incomplete cervical tSCI group (AIS grades B + C), and the SCC as the percentage of preoperative spinal canal narrowing.

Additional clinical parameters gathered at admission included patient age, sex, and comorbidity (heart disease, elevated blood pressure, and diabetes), smoking, etiology of injury, transfer from other hospitals, and the level and pattern of spinal injury according to the AOSpine subaxial cervical spine injury classification system ${ }^{28}$ (Table 2). Complications, the number of ventilator-dependent days, and overall length of stay were recorded (Table 3).

\section{Statistical Analysis}

All analyses were exploratory and performed using the $\mathrm{R}$ statistical program (R Foundation for Statistical Computing). Numerical variables were described with medians (interquartile ranges [IQRs]) and categorical variables with counts (percentages). Comparisons between groups were conducted using Mann-Whitney or Fisher exact tests, as appropriate.

An ROC curve was used to determine the optimum cutoff point for the time from injury to SD in which the maximum gain is achieved in significant neurological recovery (Youden index; the point of highest sensitivity and specificity), while 95\% confidence intervals (CIs) for cutoff point, sensitivity, and specificity were obtained by bootstrapping.

Multivariate logistic regression, double-checked using multivariate logistic regression with a Firth correction to 
TABLE 2. Demographic and injury characteristics of the patients

\begin{tabular}{|c|c|c|c|c|}
\hline Characteristic & Whole Sample & AIS Grade A $(n=41)$ & AIS Grades B + C $(n=23)$ & p Value* \\
\hline Median age (IQR), yrs & $49.0(29.5-74)$ & $42.0(25-58)$ & $74.0(46.5-78)$ & 0.001 \\
\hline Males & $51(80)$ & $35(85)$ & $16(70)$ & 0.195 \\
\hline Smoking & $17(27)$ & $12(29)$ & $5(22)$ & 0.570 \\
\hline Comorbidity & $21(33)$ & $12(29)$ & $9(39)$ & 0.580 \\
\hline Etiology of injury & & & & 0.049 \\
\hline Violence & $2(3)$ & $0(0)$ & $2(9)$ & \\
\hline Diving & $8(13)$ & $6(15)$ & $2(9)$ & \\
\hline Fall & $29(45)$ & $15(36)$ & $14(61)$ & \\
\hline MVA & $22(34)$ & $18(44)$ & $4(17)$ & \\
\hline Sport & $3(5)$ & $2(5)$ & $1(4)$ & \\
\hline Transfer from $\mathrm{RH}$ & $17(27)$ & $12(29)$ & $5(22)$ & 0.570 \\
\hline Neurological level & & & & 0.325 \\
\hline $\mathrm{C} 3$ & $1(2)$ & $1(2)$ & $0(0)$ & \\
\hline $\mathrm{C} 4$ & $6(9)$ & $6(15)$ & $0(0)$ & \\
\hline C5 & $11(17)$ & $5(12)$ & $6(26)$ & \\
\hline C6 & $20(31)$ & $13(32)$ & $7(30)$ & \\
\hline $\mathrm{C} 7$ & $21(33)$ & $13(32)$ & $8(35)$ & \\
\hline $\mathrm{C} 8$ & $5(8)$ & $3(7)$ & $2(9)$ & \\
\hline AOSpine classification & & & & 0.085 \\
\hline Type A & $6(9)$ & $2(5)$ & $4(17.5)$ & \\
\hline Type B & $7(11)$ & $3(7)$ & $4(17.5)$ & \\
\hline Type C & $51(80)$ & $36(88)$ & $15(65)$ & \\
\hline \multicolumn{5}{|l|}{ AIS grade before SD } \\
\hline$A$ & $41(64)$ & $41(100)$ & - & \\
\hline$B$ & $9(14)$ & - & $9(39)$ & \\
\hline C & $14(22)$ & - & $14(61)$ & \\
\hline Median degree of SCC (IQR) & $50.0 \%(30-51 \%)$ & $50.0 \%(40-60 \%)$ & $50.0 \%(30-50 \%)$ & 0.110 \\
\hline
\end{tabular}

account for the small sample size, was used to model significant neurological recovery with time to SD, degree of SCC, and severity of cervical tSCI. Results were considered statistically significant at the 0.05 level.

\section{Results}

\section{Study Population}

A total of 107 successive patients with tSCI and fractures or dislocations of the subaxial cervical spine were operated on during the 10-year study period. In 21 patients, surgery was not conducted within 24 hours of tSCI: in 14 due to late transfer from other hospitals, in 3 due to clear neurological recovery without compression of the spinal cord on MRI, in 2 due to polytrauma and/or comorbidity, in 1 obtunded patient due to missed tSCI at admission, and in 1 as per preference of the treating surgeon. The remaining 86 patients underwent SD within 24 hours of tSCI, but 13 of them did not meet the criteria for inclusion in the study (mostly AIS grade D). Six patients (all of them AIS grade A and over 60 years of age) who died of pulmonary failure prior to reevaluation at 6 months, 2 patients in whom the decompression was insufficient as determined by postoperative imaging, and 1 patient lost during followup were excluded from the study. Of the 64 patients who completed the study, 41 presented as AIS grade A, 9 as AIS grade $\mathrm{B}$, and 14 as AIS grade $\mathrm{C}$ injuries.

In the whole cohort, the median time to SD was 7 hours and ranged from 3 to 23 hours (Fig. 1). The median degree of SCC was $50 \%$ and ranged from $25 \%$ to $85 \%$. The difference in the time to SD and the degree of SCC between patients with complete or incomplete tSCI was not significant. Patients with complete tSCI were significantly younger (Table 2), needed longer ventilatory support, had longer hospital stays, and had a higher rate of pneumonia than patients with incomplete tSCI (Table 3). Pneumonia developed in $70.7 \%$ of patients with complete tSCI and $17.4 \%$ of patients with incomplete tSCI within 4 weeks of injury. A list of operative and perioperative characteristics and complications is provided in Table 3.

\section{Neurological Outcome by Time of SD}

The median time to SD in patients with an improvement of at least two AIS grades was 4 hours (mean $6 \pm 4.3$ 
TABLE 3. Operative and perioperative characteristics and complications

\begin{tabular}{|c|c|c|c|c|}
\hline Characteristic & Whole Sample & AIS Grade A ( $n=41)$ & AIS Grades B + C $(n=23)$ & p Value* \\
\hline Median time to SD (IQR), hrs $†$ & $7.0(5-11)$ & $7.0(6-10)$ & $6.0(4-13)$ & 0.966 \\
\hline OP procedure & & & & 0.090 \\
\hline OR + APDF & $4(6)$ & $2(5)$ & $2(9)$ & \\
\hline$C R+A D F$ & $37(58)$ & $24(58)$ & $13(56)$ & \\
\hline CR + PDF & $7(11)$ & $2(5)$ & $5(22)$ & \\
\hline CR + APDF & $16(25)$ & $13(32)$ & $3(13)$ & \\
\hline Laminectomy & $8(13)$ & $5(12)$ & $3(13)$ & $>0.999$ \\
\hline No. of fused levels & & & & 0.257 \\
\hline 1 & $22(34)$ & $11(27)$ & $11(48)$ & \\
\hline 2 & $22(34)$ & $16(39)$ & $6(26)$ & \\
\hline 3 or more & $20(31)$ & $14(34)$ & $6(26)$ & \\
\hline Fusion, at 6 mos & $64(100)$ & $41(100)$ & $23(100)$ & $>0.999$ \\
\hline \multicolumn{5}{|l|}{ Periop complications } \\
\hline Surgical infection & $3(5)$ & $3(7)$ & $0(0)$ & 0.547 \\
\hline CSF leak & $3(5)$ & $3(7)$ & $0(0)$ & 0.547 \\
\hline Cardiovascular & $6(9)$ & $6(15)$ & $0(0)$ & 0.080 \\
\hline Gastrointestinal & $5(8)$ & $3(7)$ & $2(9)$ & $>0.999$ \\
\hline Median length of $\mathrm{HS}(\mathrm{IQR})$, days & $40(26.5-60)$ & $58(37-74)$ & $29(20-35)$ & $<0.001$ \\
\hline Median ventilator ICU (IQR), days & $5(2-17.25)$ & $12(4.5-24)$ & $1(0-3)$ & $<0.001$ \\
\hline Pneumonia, first month & $33(52)$ & $29(71)$ & $4(17)$ & $<0.001$ \\
\hline
\end{tabular}

$\mathrm{ADF}=$ anterior decompression and fusion; $\mathrm{APDF}=$ anterior and posterior decompression and fusion; $\mathrm{CR}=$ closed reduction; $\mathrm{HS}=$ hospital stay; $\mathrm{OR}=$ open reduction; $\mathrm{PDF}=$ posterior decompression and fusion.

Values given as counts (\%) unless otherwise indicated.

* The $p$ values correspond to meaningful comparisons between groups AIS grade $A$ and AIS grades $B+C$ at admission.

$\dagger$ Mean and range for time to SD: whole data set $=8.8 \pm 5.2$ hours, range $3-23$ hours; AIS A $=8.4 \pm 4.3$ hours, range $3-20$ hours; AIS B $+C=$ $9.6 \pm 6.5$ hours, range $3-23$ hours.

hours). In patients with an improvement of one AIS grade or no change in AIS grade the median time to SD was 8 hours (mean $9.5 \pm 4.9$ hours). The patient whose condition deteriorated by one AIS grade underwent SD 22 hours after injury and presented with an SCC of $60 \%$.

The neurological recovery of AIS grades at 6 months following acute tSCI in regards to particular times of SD is shown in Fig. 1. The optimal time window for SD to attain a neurological improvement of at least two AIS grades was within 4 hours of injury (bootstrapped 5 hours, $95 \%$ bootstrap CI 4-9 hours). A sensitivity of 0.58 (95\% bootstrap CI 0.29-0.81) indicates how likely a patient's SD time was within that time window if there was an improvement of at least two AIS grades. A specificity of 0.92 (95\% bootstrap CI $0.83-0.98)$ indicates how likely a patient's SD time was later if there was no improvement of at least two AIS grades (Fig. 2). In our cohort, SD was performed within 4 hours of injury in 13 patients and 9 of

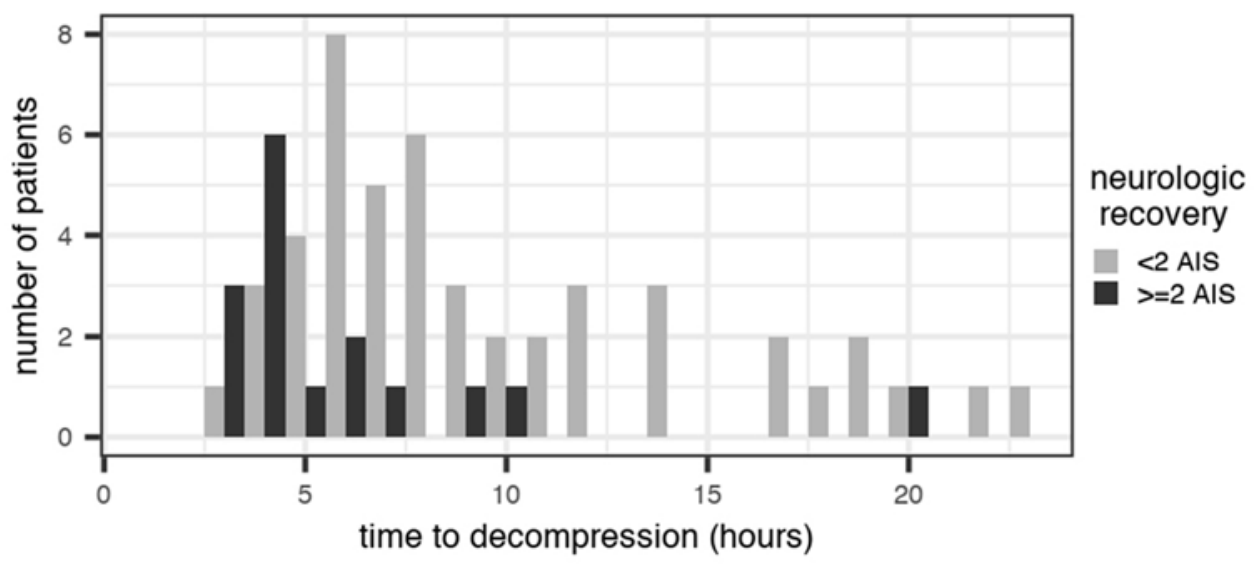

FIG. 1. Recovery of AIS grade distribution at 6 months after acute $\mathrm{tSCl}$ and SD within 24 hours. 


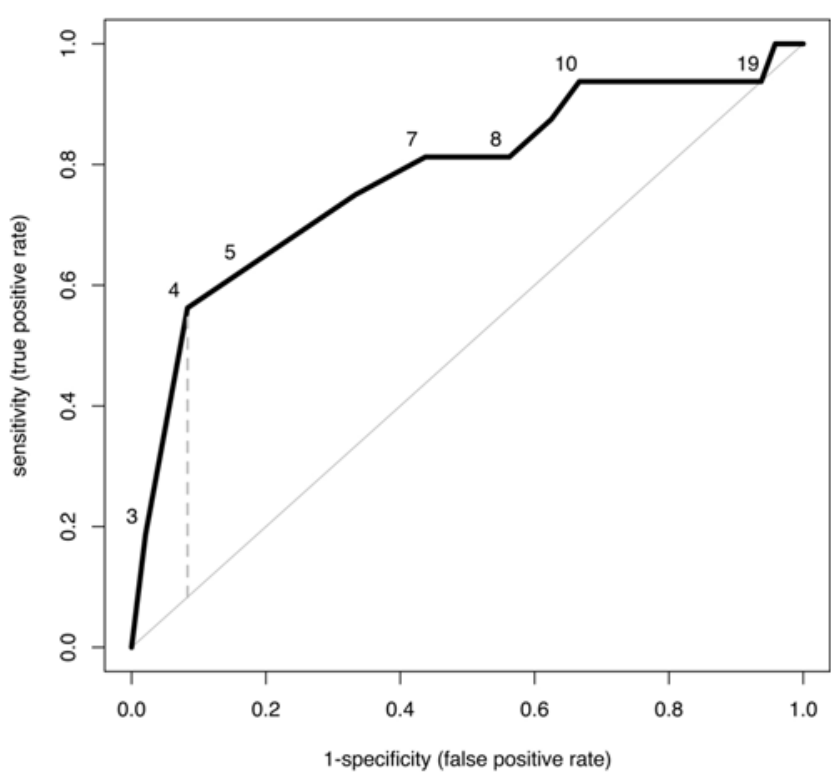

FIG. 2. ROC curve showing sensitivity against 1 - specificity of SD time in determining neurological recovery of at least two AIS grades at 6 months after tSCl. The numbers in the graph show the possible cutoff points (hours from injury to SD). The dashed gray line indicates the cutoff point of maximal gain in significant neurological recovery when operating within that time for our cohort of patients, which is 4 hours (95\% bootstrap $\mathrm{Cl} 4-9$ hours).

them attained a neurological improvement of at least two AIS grades.

\section{Association Between Significant Neurological Recovery and Time to SD, SCC, and Injury Severity}

The association between neurological recovery and time to SD, degree of SCC, and injury severity is presented in Fig. 3. Multivariate logistic regression showed that neurological recovery was statistically significantly associated with time to SD and degree of SCC (Table 4). By increasing the delay from injury to $\mathrm{SD}$, or increasing the degree of SCC before SD (controlling for other variables in the model), the chances of a neurological improvement of at least two AIS grades was reduced. There was also a tendency toward a reduced probability of neurological improvement of at least two AIS grades for AIS grade A patients, although it was not statistically significant.

The possibility that the larger the degree of SCC the shorter the time from injury to SD that is needed to obtain an improvement of at least two AIS grades was assessed by adding the interaction between time to SD and degree of SCC into a logistic model, and found to be not significant (analysis of the deviance $\mathrm{p}$ value for interaction was $0.641)$. In contrast, the predicted probabilities ${ }^{15}$ in a model allowing interaction of varying times to SD, different degrees of SCC, and completeness of injury suggested that the prognosis for recovery worsens with each additional hour before SD and each additional percentage of SCC (Fig. 4).

\section{Discussion}

In the present prospective cohort study at a single trauma center, we found that the first 4-9 hours of cervical tSCI represents a window of opportunity for SD with the best chances for neurological recovery entailing at least two AIS grades 6 months after injury. In addition to the SD timing, the degree of SCC before SD was a significant independent predictor of neurological recovery. There was also a tendency toward a reduced probability of neurological recovery in patients who presented with complete versus incomplete cervical tSCI.

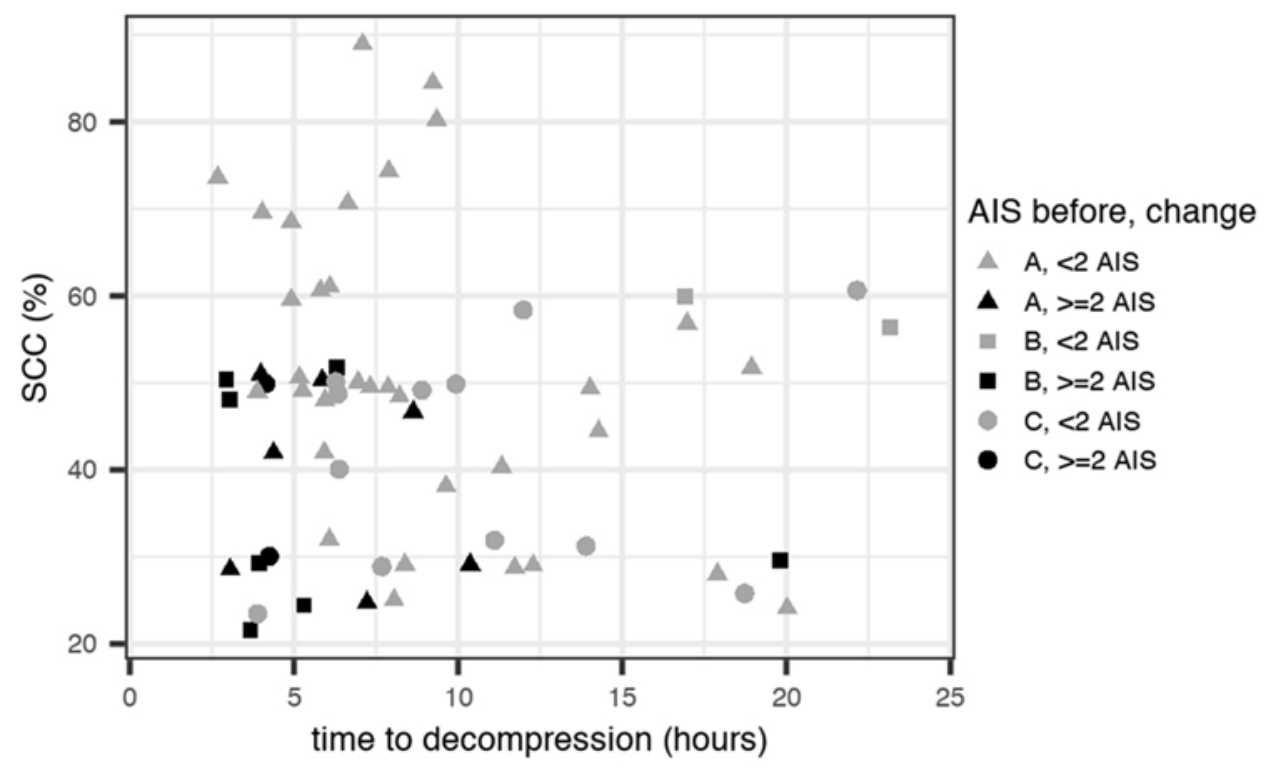

FIG. 3. Time to SD, degree of SCC, and severity of injury at admission of all patients and the change in their AIS grade after 6 months. To avoid complete overlap, the points are slightly jittered. 
TABLE 4. Association between significant neurological recovery and time to SD, degree of SCC, and injury severity in three multivariate logistic regression models

\begin{tabular}{|c|c|c|c|c|c|c|c|c|c|}
\hline \multirow[b]{2}{*}{ Variable } & \multicolumn{3}{|c|}{ Whole Cohort } & \multicolumn{3}{|c|}{ AIS Grade A } & \multicolumn{3}{|c|}{ AIS Grades B + C } \\
\hline & Estimate & SE & $p$ Value & Estimate & SE & p Value & Estimate & SE & $\mathrm{p}$ Value \\
\hline Regression constant & 3.65 & 1.70 & 0.03 & 5.98 & 2.87 & 0.04 & 2.82 & 1.99 & 0.16 \\
\hline AIS grade $B$ or $C$ (A referent) & 0.88 & 0.71 & 0.21 & - & - & - & - & - & - \\
\hline Time to SD & -0.24 & 0.11 & 0.01 & -0.42 & 0.22 & 0.06 & -0.22 & 0.12 & 0.07 \\
\hline SCC & -0.07 & 0.03 & 0.02 & -0.10 & 0.04 & 0.02 & -0.04 & 0.04 & 0.39 \\
\hline
\end{tabular}

$\mathrm{SE}=$ standard error

We do not report the results of multivariate logistic regression with a Firth correction because they do not alter the conclusions.

\section{Neurological Outcome According to Time of SD}

Our finding is consistent with our previous observations of a higher rate of neurological improvement of at least two AIS grades at 6 months after acute cervical tSCI in patients operated on within the first 8 hours of injury compared to 8-24 hours after injury. ${ }^{18}$ In contrast, in a recent multicenter retrospective study, a lower rate of neurological improvement by at least two AIS grades after at least 6 months of follow-up was reported in patients with acute cervical tSCI operated on within the first 5 hours of injury compared to 5-24 hours after injury. ${ }^{20}$ However, in that study the higher rate of patients with complete tSCI allocated to the 5-hour group and the relatively short time (8.2 \pm 5.9 hours) from injury to SD in the 5- to 24 -hour group may have significantly influenced the neurological outcome in favor of the 5- to 24-hour group.

\section{Association Between Neurological Recovery and Time to SD, SCC, and Injury Severity}

In the present study, we found that, in addition to the time to SD, the degree of SCC was a significant predictor of neurological recovery. Notably, no patients with persistent SCC degrees of more than $60 \%$ before SD recovered any AIS grade, even if SD was achieved within 4 hours of injury, and patients with SCC degrees of approximately $50 \%$ had a significant neurological recovery only if they had undergone SD within 6 hours of injury. Conversely, some patients with minor degrees of SCC recovered sig-

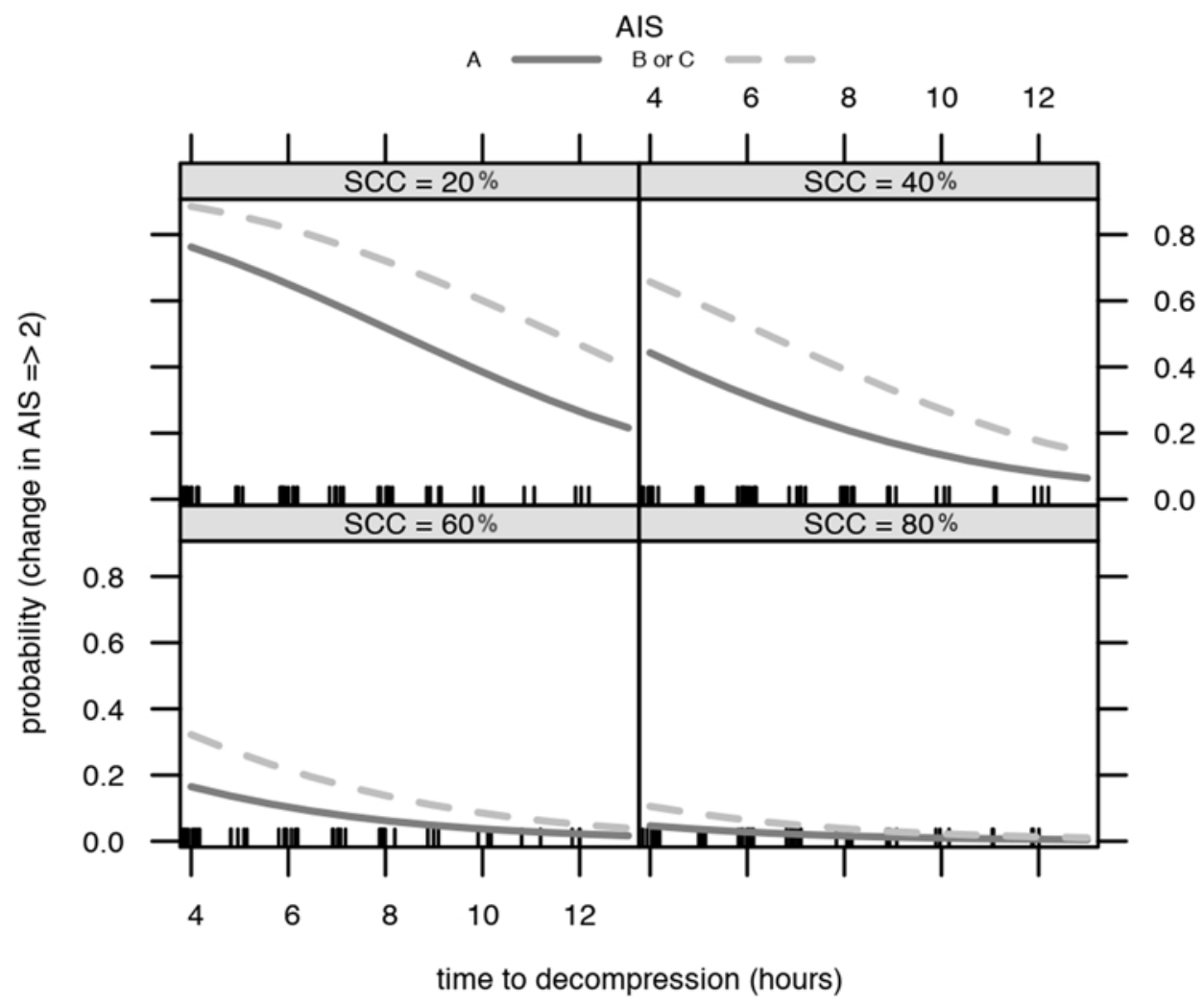

FIG. 4. Predicted probabilities in a model of different times to SD, degrees of SCC, and injury severity (AIS grade A vs AIS grades $\mathrm{B}+\mathrm{C})$ for prognosis. 
nificantly even if SD was performed at later time points. Accordingly, intramedullary lesion expansion in cervical tSCI, which was shown to be positively related to the degree of SCC, may be mitigated by early SD. ${ }^{1,2}$ Surprisingly, by increasing both the time to SD and the degree of SCC, we found no negative potentiation to reduce the probability of neurological recovery in our patients. Although this might be a result of the small sample size, it is also possible there might be an upper limit on the degree of tolerable SCC after which the timing of SD could not significantly influence the neurological recovery. As indicated by preclinical studies, ${ }^{4,8-10,27}$ a poor neurological outcome in patients with tSCI and a high degree of SCC might be a consequence of hypoperfusion at the injury site due to high compression force on the spinal cord.

The severity of primary injury plays a significant role in the pathophysiology of tSCI in preclinical models. ${ }^{21}$ Therefore, the severity of primary injury might also be an important predictor of neurological recovery in patients with cervical tSCI irrespective of the degree of SCC or timing of SD. Although the severity of primary injury may not be directly reflected in AIS grades, more prominent posttraumatic changes on MRI were reported in clinical studies comparing patients with complete tSCI with patients with incomplete tSCI at admission..$^{22,24,26}$ In our cohort, only $17.5 \%$ of patients with complete tSCI compared to $39.7 \%$ of patients with incomplete tSCI recovered at least two AIS grades, which was not significantly different. However, if SCC was eliminated from the multivariate analysis, completeness of injury at admission resulted in a significant negative predictor of neurological recovery. Of note, $50 \%$ of AIS grade A patients with SD within 4 hours of injury recovered at least two AIS grades compared to $11.4 \%$ of AIS grade A patients with later SD, which might suggest that ultra-early SD may have beneficially influenced the neurological outcome in some AIS grade A patients. Accordingly, the AIS grade before decompression had no predictive value if closed reduction and decompression after tSCI due to cervical facet dislocation in football players was achieved within 4 hours of injury in contrast to later time windows. ${ }^{23}$ In contrast, a higher rate of pneumonia found in our study in AIS grade A patients may have had a negative impact on neurological outcome in these patients, as recently suggested. ${ }^{17}$ In addition, patients with complete tSCI in our study needed longer ventilatory support and had a longer hospital stay. In our view, these complications are strongly related to the severity of primary cervical tSCI, and thus cannot be assessed as an independent factor of neurological recovery, although they may contribute.

Yet, we can assume there are patients whose severe primary spinal cord injury and/or high degree of SCC make them unresponsive for significant neurological recovery even to ultra-early SD, with degrees of SCC higher than $60 \%$ consistent with complete cervical tSCI at admission and the follow-up in our cohort. Moreover, SCC was found to be a significant negative predictor of neurological recovery in patients with complete tSCI but not in patients with incomplete tSCI in our cohort. This may be due to more prominent local swelling of the spinal cord reported in patients with complete compared to patients with in- complete $\mathrm{tSCI},{ }^{22,24,26}$ which is likely to result in a comparatively greater compression force on the damaged spinal cord at the same degree of SCC, as shown in preclinical rodent models..$^{21}$ Clinical trials showed that higher intraspinal pressure and lower spinal cord perfusion pressure at the site of tSCI negatively predict neurological outcome after injury. ${ }^{25}$ Together, our results suggest that significant neurological recovery in patients with tSCI depends not only on the time of SD but also on injury severity and the degree of SCC, and these three factors should probably not be evaluated independently. However, because our results pertain to patients with severe tSCI (i.e., those with AIS grades A-C), they may not be applicable to patients with AIS grade D. Further studies are required to better understand and determine the interrelationships among injury severity, degree of SCC, and time of SD, and their effect on neurological outcome in tSCI.

\section{Study Limitations}

We are aware that a study involving 64 patients may lack statistical power, especially in light of cervical tSCI heterogeneity. To minimize this effect, we set narrow inclusion criteria and provided a uniform treatment protocol in the same institution for all patients because minor discrepancies between treatment protocols may have unpredicted consequences. In this regard, we believe that because our study was performed in a single center with all included patients treated by the same surgical team, in the same ICU and in the same rehabilitation institution, with the same acute treatment and rehabilitation protocols, it offers reliable data. And, despite the modest number of patients, the study may have some advantages over multicenter studies. We also believe there might be other factors involved in determining secondary injury severity besides the completeness of cervical tSCI and the degree and duration of spinal cord compression, with one factor being the energy of primary injury. Yet, we found no specific method to objectively evaluate the energy of the impact at the site of cervical tSCI in humans. In addition, we are aware of the possibility of inaccuracy of the neurological examination at admission, particularly in patients in spinal shock. We tried to address this by a second examination 24 hours after injury, but $70 \%$ of patients needed ventilatory support and were sedated and intubated for at least 72 hours after the operation. Therefore, the second neurological examination 24 hours after SD proved unreliable and was not incorporated in the study. Moreover, because spinal shock occurs in almost all patients with severe cervical tSCI, starting within minutes and lasting up to 12 months after injury, ${ }^{11}$ a second examination would not have overcome this limitation.

\section{Conclusions}

Our findings indicate that the impact of SD within the first 24 hours following acute cervical tSCI on neurological recovery is time dependent and that the optimal time window for SD is within the first 4-9 hours of injury, depending on the degree of SCC and severity of injury. The higher the severity of injury and the degree of SCC, the shorter is the time window of opportunity from injury to 
SD for significant neurological recovery, but other factors may contribute as well. Further studies are required to better understand the interrelationship between the timing of $\mathrm{SD}$, severity of injury, degree of SCC, and other factors, and its effect on neurological outcome in patients with cervical tSCI.

\section{Acknowledgments}

We thank Uroš Tominc for assistance in data collection. This study was financially supported by the Ministry of Health of the Republic of Slovenia, and by the Slovenian Research Agency (grant nos. P3-0338 and P3-0154).

\section{References}

1. Aarabi B, Sansur CA, Ibrahimi DM, Simard JM, Hersh DS, Le E, et al: Intramedullary lesion length on postoperative magnetic resonance imaging is a strong predictor of ASIA impairment scale grade conversion following decompressive surgery in cervical spinal cord injury. Neurosurgery 80:610-620, 2017

2. Aarabi B, Simard JM, Kufera JA, Alexander M, Zacherl $\mathrm{KM}$, Mirvis SE, et al: Intramedullary lesion expansion on magnetic resonance imaging in patients with motor complete cervical spinal cord injury. J Neurosurg Spine 17:243-250, 2012

3. Ahuja CS, Wilson JR, Nori S, Kotter MRN, Druschel C, Curt A, et al: Traumatic spinal cord injury. Nat Rev Dis Primers 3:17018, 2017

4. Batchelor PE, Wills TE, Skeers P, Battistuzzo CR, Macleod MR, Howells DW, et al: Meta-analysis of pre-clinical studies of early decompression in acute spinal cord injury: a battle of time and pressure. PLoS One 8:e72659, 2013

5. Bracken MB, Shepard MJ, Holford TR, Leo-Summers L, Aldrich EF, Fazl M, et al: Administration of methylprednisolone for 24 or 48 hours or tirilazad mesylate for 48 hours in the treatment of acute spinal cord injury. Results of the Third National Acute Spinal Cord Injury Randomized Controlled Trial. National Acute Spinal Cord Injury Study. JAMA 277:1597-1604, 1997

6. Bracken MB, Shepard MJ, Holford TR, Leo-Summers L, Aldrich EF, Fazl M, et al: Methylprednisolone or tirilazad mesylate administration after acute spinal cord injury: 1-year follow up. Results of the third National Acute Spinal Cord Injury randomized controlled trial. J Neurosurg 89:699-706, 1998

7. Branco F, Cardenas DD, Svircev JN: Spinal cord injury: a comprehensive review. Phys Med Rehabil Clin N Am 18:651-679, v, 2007

8. Carlson GD, Minato Y, Okada A, Gorden CD, Warden KE, Barbeau JM, et al: Early time-dependent decompression for spinal cord injury: vascular mechanisms of recovery. J Neurotrauma 14:951-962, 1997

9. Carlson GD, Warden KE, Barbeau JM, Bahniuk E, KutinaNelson KL, Biro CL, et al: Viscoelastic relaxation and regional blood flow response to spinal cord compression and decompression. Spine (Phila Pa 1976) 22:1285-1291, 1997

10. Dimar JR II, Glassman SD, Raque GH, Zhang YP, Shields $\mathrm{CB}$ : The influence of spinal canal narrowing and timing of decompression on neurologic recovery after spinal cord contusion in a rat model. Spine (Phila Pa 1976) 24:1623-1633, 1999

11. Ditunno JF, Little JW, Tessler A, Burns AS: Spinal shock revisited: a four-phase model. Spinal Cord 42:383-395, 2004

12. Fehlings MG, Rao SC, Tator CH, Skaf G, Arnold P, Benzel E, et al: The optimal radiologic method for assessing spinal canal compromise and cord compression in patients with cervical spinal cord injury. Part II: Results of a multicenter study. Spine (Phila Pa 1976) 24:605-613, 1999

13. Fehlings MG, Tetreault LA, Wilson JR, Aarabi B, Anderson P, Arnold PM, et al: A clinical practice guideline for the management of patients with acute spinal cord injury and central cord syndrome: recommendations on the timing $(\leq 24$ hours versus $>24$ hours) of decompressive surgery. Global Spine J 7 (3 Suppl):195S-202S, 2017

14. Fehlings MG, Vaccaro A, Wilson JR, Singh A, Cadotte DW, Harrop JS, et al: Early versus delayed decompression for traumatic cervical spinal cord injury: results of the Surgical Timing in Acute Spinal Cord Injury Study (STASCIS). PLoS One 7:e32037, 2012

15. Fox J: Effect displays in R for generalised linear models. J Stat Softw 8:1-27, 2003

16. Geisler FH, Coleman WP, Grieco G, Poonian D: The Sygen multicenter acute spinal cord injury study. Spine (Phila Pa 1976) 26 (24 Suppl):S87-S98, 2001

17. Jaja B, Jiang F, Badhiwala JH, Schär R, Kurpad S, Grossman $\mathrm{RG}$, et al: Association of pneumonia, wound infection, and sepsis with clinical outcomes after acute traumatic spinal cord injury. J Neurotrauma 36:3044-3050, 2019

18. Jug M, Kejžar N, Vesel M, Al Mawed S, Dobravec M, Herman S, et al: Neurological recovery after traumatic cervical spinal cord injury is superior if surgical decompression and instrumented fusion are performed within 8 hours versus 8 to 24 hours after injury: a single center experience. J Neurotrauma 32:1385-1392, 2015

19. Kirshblum SC, Burns SP, Biering-Sorensen F, Donovan W, Graves DE, Jha A, et al: International standards for neurological classification of spinal cord injury (revised 2011). J Spinal Cord Med 34:535-546, 2011

20. Mattiassich G, Gollwitzer M, Gaderer F, Blocher M, Osti M, Lill M, et al: Functional outcomes in individuals undergoing very early $(<5 \mathrm{~h})$ and early (5-24 h) surgical decompression in traumatic cervical spinal cord injury: analysis of neurological improvement from the Austrian Spinal Cord Injury Study. J Neurotrauma 34:3362-3371, 2017

21. Mihai G, Nout YS, Tovar CA, Miller BA, Schmalbrock P, Bresnahan JC, et al: Longitudinal comparison of two severities of unilateral cervical spinal cord injury using magnetic resonance imaging in rats. J Neurotrauma 25:1-18, 2008

22. Miyanji F, Furlan JC, Aarabi B, Arnold PM, Fehlings MG: Acute cervical traumatic spinal cord injury: MR imaging findings correlated with neurologic outcome-prospective study with 100 consecutive patients. Radiology 243:820827,2007

23. Newton D, England M, Doll H, Gardner BP: The case for early treatment of dislocations of the cervical spine with cord involvement sustained playing rugby. J Bone Joint Surg Br 93:1646-1652, 2011

24. Rutges JPHJ, Kwon BK, Heran M, Ailon T, Street JT, Dvorak MF: A prospective serial MRI study following acute traumatic cervical spinal cord injury. Eur Spine J 26:2324-2332, 2017

25. Saadoun S, Chen S, Papadopoulos MC: Intraspinal pressure and spinal cord perfusion pressure predict neurological outcome after traumatic spinal cord injury. J Neurol Neurosurg Psychiatry 88:452-453, 2017

26. Shabani S, Kaushal M, Budde M, Kurpad SN: Correlation of magnetic resonance diffusion tensor imaging parameters with American Spinal Injury Association score for prognostication and long-term outcomes. Neurosurg Focus 46(3):E2, 2019

27. Streijger F, So K, Manouchehri N, Tigchelaar S, Lee JHT, Okon EB, et al: Changes in pressure, hemodynamics, and metabolism within the spinal cord during the first 7 days after injury using a porcine model. J Neurotrauma 34:33363350,2017 
28. Vaccaro AR, Koerner JD, Radcliff KE, Oner FC, Reinhold M, Schnake KJ, et al: AOSpine subaxial cervical spine injury classification system. Eur Spine J 25:2173-2184, 2016

\section{Disclosures}

The authors report no conflict of interest concerning the materials or methods used in this study or the findings specified in this paper.

\section{Author Contributions}

Conception and design: Jug, Kejžar, Bajrović. Acquisition of data: Jug. Analysis and interpretation of data: Jug, Kejžar, Bajrović. Drafting the article: Jug, Bajrović. Critically revising the article: all authors. Reviewed submitted version of manuscript: Jug, Kejžar, Bajrović. Approved the final version of the manuscript on behalf of all authors: Jug. Statistical analysis: Kejžar. Administrative/technical/material support: Jug, Cimerman. Study supervision: Jug, Cimerman, Bajrović.

\section{Supplemental Information}

Previous Presentations

Parts of this work were presented as proceedings at the Annual Spine Experts Group Meeting and Slovenia 2017 Spine Symposium (Ljubljana, Slovenia, November 2017), at Eurospine 2018 (Barcelona, Spain, September 2018), and at the Global Spine Congress 2019 (Toronto, ON, Canada, May 2019).

\section{Correspondence}

Marko Jug: University Medical Centre Ljubljana, Ljubljana, Slovenia.jugmarko74@gmail.com. 